\title{
Talo-patello-scaphoid osteolysis
}

INSERM

\section{Source}

INSERM. (1999). Orphanet: an online rare disease and orphan drug data base. Talopatello-scaphoid osteolysis. ORPHA:50809

Talo-patello-scaphoid osteolysis is an extremely rare form of primary osteolysis (see this term), described in two sisters to date, characterized by bilateral osteolysis of the tali, scaphoids, and patellae (accompanied by periarticular swelling and pain) and short fourth metacarpals (brachydactyly type E; see this term), in the absence of renal disease.

Autosomal recessive inheritance has been suggested. 